\title{
Phenobarbitone induced drug reaction with eosinophilia and systemic symptoms (DRESS): a case report
}

\author{
Navreet K. Natt ${ }^{1}$, Tarsem Singh ${ }^{2}$, Harmanjit Singh ${ }^{3}$, Manu Sharma ${ }^{2}$, Gagandeep Singh ${ }^{2}$
}

\author{
${ }^{1}$ Medical officer, CHC Manawala, Amritsar, India \\ ${ }^{2}$ Department of Paediatrics, Sri Guru Ram Das \\ Institute of Medical Sciences and Research, Vallah, \\ Amritsar, India \\ ${ }^{3}$ Department of Pharmacology, PGIMER, \\ Chandigarh, India
}

Received: 9 March 2013

Revised: 21 March 2013

Accepted: 8 April 2013

\section{*Correspondence to:}

Dr. Navreet K. Natt,

Email: navreet_natt@yahoo.co.in

(C) 2013 Natt NK et al. This is an open-access article distributed under the terms of the Creative Commons Attribution License, which permits unrestricted use, distribution, and reproduction in any medium, provided the original work is properly cited.

\begin{abstract}
Drug reaction with eosinophilia and systemic symptoms (DRESS) is a life threatening cutaneous drug reaction with visceral involvement and hematological abnormalities. Being a rare side effect, it is often under-reported and misdiagnosed. The fatal adverse drug reaction is associated most commonly with aromatic anti-epileptics phenytoin, carbamazepine and less frequently with phenobarbitone. Here, we report a case of phenobarbitone induced DRESS in a 1 year old male child. He succumbed to fulminant hepatic failure inspite of being put on steroids, hepatoprotectives, antibiotics and ventilatory support.
\end{abstract}

Keywords: Phenobarbitone, Adverse drug reactions, Antiepileptics

\section{INTRODUCTION}

Several cutaneous reactions known as SCAR: severe cutaneous adverse reactions to drugs, occur after drug exposure manifesting with variable signs-symptoms and time interval. Drug reaction with eosinophilia and systemic symptoms (DRESS) also known as DIHS (druginduced hypersensitivity syndrome) is an underrecognized and potentially life threatening drug reaction characterized by fever, skin rash, hematological abnormalities including eosinophilia, leukocytosis or atypical lymphocytes with systemic involvement including hepatitis, interstitial nephritis or pneumonitis. DRESS is a rare side effect of aromatic anticonvulsive drugs (e.g. phenobarbitone, phenytoin and carbamazepine). Phenobarbitone is rarely implicated for the syndrome; carbamazepine and phenytoin being more common causative agents. ${ }^{1}$ Other drugs like allopurinol, sulfasalazine, lamotrigine, valproic acid, nonsteroidal anti-inflammatory drugs, nevirapine and vancomycin have also been associated with this syndrome. ${ }^{2}$
DIHS was originally described in 1950 by Chaiken et al as a triad of fever, rash, and multi-organ failure and the acronym DRESS was then put forth much later in 1996 by Bocquet et al. ${ }^{3,4}$ The overall mortality is $10 \%$, but can be up to $40 \%$ in presence of organ failure. ${ }^{5}$ Thus, timely recognition of this potentially fatal drug reaction is required to prevent the sequelae.

Here we present a case report of a 1-year old male child who developed DRESS after taking phenobarbitone. He was put on steroids, antibiotics, hepatoprotectives and ventilated but he ultimately succumbed to fatal sequelae of fulminant hepatic failure.

\section{CASE REPORT}

A one year old male child presented with history of an episode of generalized tonic-clonic seizures 30 days back, generalized rash since 15 days, drowsiness and difficulty in breathing since 3 days. He had been put on phenobarbitone (PHB) for GTCS since 30 days. On history taking the parents reported that rash had started 
on face, progressed to extremities and then to abdomen and trunk. His past history was unremarkable. No history of blood transfusion, surgery, pertussis, measles or contact with tuberculosis was present. Review of systems was positive for fever, high-coloured urine, shortness of breath, altered sensorium and negative for chest pain, abdominal pain, nausea, vomiting, and weight-loss.

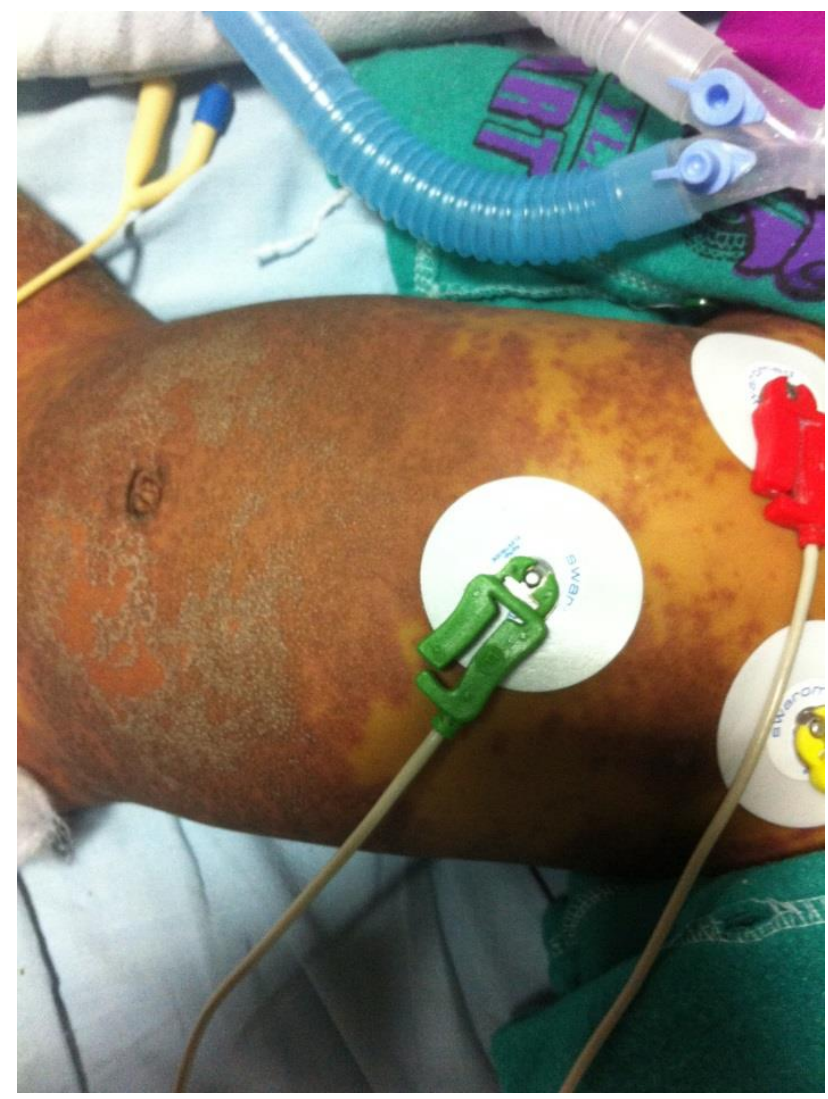

Figure 1: Rash on the abdomen, trunk and extremities of a one year old male child with DRESS (drug reaction with eosinophilia and systemic symptoms) caused by phenobarbitone.

On examination temperature was $39^{\circ} \mathrm{C}$, pulse 134 per minute and respiratory rate 40 per minute. The child was toxic, icteric with tachypnea and tender hepatomegaly. He had an altered sensorium with GCS 10/15. The rash was generalized maculopapular showing scaling without vesiculation or blistering. At this point the differential diagnosis considered were drug induced hypersensitivity, meningococcal meningitis, autoimmune or viral hepatitis, Stevens - Johnson syndrome, scarlet fever.

Laboratory results revealed a white blood cell count of 38 thousand $/ \mathrm{mm}^{3}$ (normal from 4.0 to 10.0 thousand $/ \mathrm{mm}^{3}$ ), with $49 \%$ neutrophils, $38 \%$ lymphocytes, and $5.0 \%$

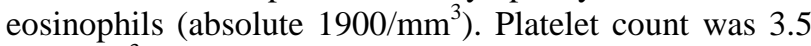
lakh/mm $\mathrm{mm}^{3}$. Hepatic function panel revealed transaminitis i.e. an aspartate aminotransferase of 1850U/L (normal from 0 to 37), and alanine aminotransferase (ALT) of $1410 \mathrm{U} / \mathrm{L}$ (normal from 0 to 41). Evaluation for acute and chronic hepatitis with serologies was negative for hepatitis A, B, and C. Reports of CSF cytology, biochemistry and blood culture were unremarkable. Chest $\mathrm{X}$-Ray was normal.

Hepatic function was the main concern. The child was admitted in paediatric intensive care unit. PHB was stopped and he was put on steroids, hepatoprotective agents, antibiotics, antihistamines. The child's condition did not improve inspite of this. Dyspnoea worsened with cold extremities and weak pulses. Transaminitis further increased and GCS deteriorated to 7/15 on second day. The child was ventilated and vasopressors were added but there was no improvement. On third day, the child succumbed to sequelae of hepatic failure i.e. shock and cardiac failure.

\section{DISCUSSION}

The patient is presented as a case of DRESS due to antiepileptic drug Phenobarbitone. PHB although less commonly used in developed countries due its adverse drug reactions, is being widely used in developing countries due to its low cost and efficacy. PHB is still considered a safe drug especially in paediatric age group. Anticonvulsant drugs, especially those with an aromatic ring structure such as carbamazepine, phenytoin, and phenobarbitone cause DRESS syndrome, incidence being 1 in 1000 to 1 in 10,000 and fatality rate being $10 \%$ amongst the cases. ${ }^{6}$

DRESS is an under-diagnosed type IV (delayed type) hypersensitivity reaction which occurs 2 to 8 weeks after initiation of offending drug. The diagnosis of DRESS syndrome involves three criterias ${ }^{7}$ :

1. Widespread rash mostly maculopapular in nature, erythroderma and pustules may be present.

2. Eosinophilia, Absolute Eosinophil count $>1500 / \mathrm{dL}$, or atypical lymphocytes, leukocytosis.

3. Internal organ involvement: at least one of these i.e. hepatitis, interstitial nephropathy, interstitial lung disease, myocardial involvement, lymphadenopathy.

In order to evaluate potential cases of DRESS, a RegiSCAR (Severe Cutaneous Adverse Reactions) criteria $^{8}$ was designed in 2007 study group which published a scoring system with 8 diagnostic criterias: first, fever greater than $38.5^{\circ} \mathrm{C}$; second, enlarged lymph nodes; third, eosinophilia; fourth, atypical lymphocytosis; fifth, skin involvement; sixth, organ involvement; seventh, resolution greater than 15 days; and eighth, evaluation and exclusion of other causes. The score indicates likelihood of DRESS:

\section{Score $<2$ indicates no DRESS}

Score 2 to 3 indicates possible case

Score 4 to 5 indicates probable case

Score $>5$ indicates definite case

The patient in this case report had a score of six points (two each for eosinophilia and skin rash suggestive of 
DRESS, one each for liver involvement, and evaluation of other potential causes, indicating the case as 'definite' as per the RegiSCAR scoring guidelines.

The mechanisms of DRESS though not completely understood, but pharmacogenetic and immunologic mechanism is involved. The aromatic anticonvulsants are oxidized to arene oxides by the cytochrome p-450 system. These arene oxides are toxic to cells and cause cell death and immunological response. Epoxide hydroxylases detoxify these arene oxides but in some individuals these detoxifying enzyme systems may be absent or mutated which can contribute to DRESS due to accumulation of toxic metabolites like arene oxides. ${ }^{9}$ Recent studies suggest that reactivation of HHV-6 may be a contributing factor in pathogenesis. Due to likelihood of genetic predisposition, first degree relatives should be cautioned about the drugs. ${ }^{9}$

DRESS has a favorable outcome if recognized early and the offending drug is stopped before significant visceral involvement. The most important determinant of prognosis of DRESS is hepatitis and subsequent hepatic failure. This fact necessitates the need for continuing liver function tests. Hepatoprotective agents can be given to improve liver function. Systemic corticosteroids have become a mainstay of treatment of DRESS. Intravenous immunoglobulin (IVIG) and/or plasmapheresis are other modalities of treatment if the condition deteriorates. Recovery is usually slow and may take weeks to months. ${ }^{10}$

\section{CONCLUSION}

Taking into consideration the high mortality rate with DRESS and its association with most commonly prescribed anti-epileptics, clinicians should be aware of this life threatening syndrome while starting any new antiepileptic drug. DRESS should be considered as differential diagnosis in patients presenting with skin rash and systemic abnormalities. Lives can be saved by early recognition of DRESS and prompt removal of offending agent.

Funding: No funding sources Conflict of interest: None declared

Ethical approval: Not required

\section{REFERENCES}

1. Yang CY, Dao RL, Lee TJ, Lu CW, Yang CH, Hung SI, et al. Severe cutaneous adverse reactions to antiepileptic drugs in Asians. Neurology 2011;77:2025-33.

2. Krauss G. Current understanding of delayed anticonvulsant hypersensitivity reactions. Epilepsy Curr 2006;6:33-7.

3. Chaiken BH, Goldberg BI, Segal JP. Dilantin sensitivity: report of a case of hepatitis with jaundice, pyrexia and exfoliative dermatitis. $\mathrm{N}$ Engl J Med 1950;242:897-8.

4. Bocquet H, Bagot M, Roujeau JC. Drug-induced pseudolymphoma and drug hypersensitivity syndrome (Drug Rash with Eosinophilia and Systemic Symptoms: DRESS). Semin Cutan Med Surg 1996;15:250-7.

5. Tas S, Simonart T. Management of drug rash with eosinophilia and systemic symptoms (DRESS syndrome): an update. Dermatology 2003;206:3536.

6. Gennis MA, Vemuri R, Burns EA, et al. Familial occurrence of hypersensitivity to phenytoin. Am J Med 1991;91:631-4.

7. Vittorio CC, Muglia JJ. Anticonvulsant hypersensitivity syndrome. Arch Intern Med 1995; 155:2285-90.

8. Kardaun SH, Sidoroff A, Valeyrie-Allanore L, Halevy S, Davidovici BB, Mockenhaupt M, et al. Variability in the clinical pattern of cutaneous sideeffects of drugs with systemic symptoms: does a DRESS syndrome really exist? $\mathrm{Br} \mathrm{J}$ Dermatol 2007;156:609-11.

9. Kim CW, Choi GS, Yun CH, Kim DI. Drug hypersensitivity to previously tolerated phenytoin by carbamazepine-induced DRESS syndrome. J Korean Med Sci 2006;21:768-72.

10. Criado PR, Criado RF, Avancini Jde M, Santi CG. Drug reaction with Eosinophilia and Systemic Symptoms (DRESS) / Drug-induced Hypersensitivity Syndrome (DIHS): a review of current concepts. An Bras Dermatol 2012;87:43549.

doi:10.5455/2319-2003.ijbcp20130620

Cite this article as: Natt NK, Singh $\mathrm{T}$, Singh $\mathrm{H}$,

Sharma M, Singh G. Phenobarbitone induced drug reaction with eosinophilia and systemic symptoms (DRESS): a case report. Int J Basic Clin Pharmacol 2013;2:333-5. 